\title{
Aloimunização eritrocitária em pacientes politransfundidos com doenças onco- hematológicas: Uma revisão de literatura
}

Erythrocyte alloimmunization in polytransfused patients with onco-hematological diseases: A literature review

Aloinmunización de eritrocitosen pacientes politransfundidos con enfermedades oncohematológicas: Revisión de la literatura

\section{Resumo}

O objetivo desta revisão de literatura foi avaliar quais são as doenças onco-hematológicas mais frequentes em pacientes politransfundidos aloimunizados, determinar quais são os antígenos eritrocitários e os aloanticorpos antieritrocitários mais comuns detectados no perfil populacional do estudo, assim como identificar medidas adequadas a serem tomadas a fim de minimizar as taxas de aloimunização nesta população dependente de transfusão. Foram utilizados critérios de elegibilidade bem delineados e a busca ocorreu na base de dados bibliográfica eletrônica Medical Literature Analysis and Retrieval System Online (MEDLINE). A partir dos critérios utilizados foram incluídos 10 artigos para compor esta revisão.Verificou-seque a doença onco-hematológica mais prevalente na população estudada foi a Síndrome Mielodisplásica e os aloanticorpos detectados com maior frequência foram os associados aos sistemas Rh, Kell, MNS e Kidd. Todos os trabalhos apontaram a fenotipagem eritrocitária extensa como forma de minimizar as taxas de aloimunização entre os pacientes com neoplasias hematológicas, assim como reduzir os riscos de ocorrência de reações hemolíticas transfusionais. Portanto, a realização da fenotipagem e genotipagem para a identificação de outros sistemas além do $\mathrm{ABO} / \mathrm{Rh}$, principalmente para os pacientes cronicamente transfundidos e que apresentam Síndrome Mielodisplásica, pode minimizar o risco de aloimunização e suas consequências com reflexos positivos na segurança e eficácia da terapia transfusional.

Palavras-chave: Aloimunização; Anticorpo irregular; Fenotipagem eritrocitária; Doenças onco-hematológicas; Politransfusão.

\footnotetext{
Abstract

The purpose of this literature review was to evaluate which onco-hematologic diseases are most frequent in alloimmunized polytransfused patients, to determine which are the most common erythrocyte antigens and antierythrocyte alloantibodies detected in the study population profile, and to identify appropriate measures to be taken to minimize alloimmunization rates in this transfusion-dependent population. Well-defined eligibility criteria were used, and the search was conducted in the electronic bibliographic data base Medical Literature Analysis and Retrieval System Online (MEDLINE). Based on the criteria used, 10 articles were included in this review. It was found that the most prevalent onco-hematologic disease in the population studied was Myelodysplastic Syndrome and the most frequently detected alloantibodies were those associated with the Rh, Kell, MNS and Kidd systems. All the papers pointed to extensive erythrocyte phenotyping to minimize alloimmunization rates among patients with hematological neoplasms, as well as to reduce the risks of occurrence of transfusion hemolytic reactions. Therefore, performing phenotyping and genotyping to identify systems other than $\mathrm{ABO} / \mathrm{Rh}$, especially for chronically transfused patients who present with Myelodysplastic Syndrome, can minimize the risk of alloimmunization and its consequences with positive reflections on the safety and efficacy of transfusion therapy.
} 
Keywords: Alloimmunization; Irregular antibody; Erythocyte phenotyping; Onco-hematological diseases; Multitransfused patients.

\section{Resumen}

El objetivo de esta revisión de la literatura fue evaluar las enfermedades onco-hematológicas más frecuentes en pacientes politransfundidos aloinmunizados, para determinar cuáles son los antígenos eritrocitarios y los aloanticuerpos antieritrocitos más comunes detectados en el perfil de la población de estudio, así como identificar las medidas adecuadas a ser tomado a fin de minimizar las tasas de aloinmunización en esta población dependiente de transfusiones. Se utilizaron criterios de elegibilidad bien definidos y la búsqueda se realizó en la base de datos bibliográfica electrónica Medical Literature Analysis and Retrieval System Online (MEDLINE). Con base en los criterios utilizados, se incluyeron 10 artículos para componer esta revisión. Se encontró que la enfermedad oncohematológica más prevalente en la población estudiada fue el Síndrome Mielodisplásico y los aloanticuerpos detectados con mayor frecuencia fueron los asociados a Rh, Kell, MNS. y sistemas Kidd. Todos los estudios señalaron la fenotipificación extensa de eritrocitos como una forma de minimizar las tasas de aloinmunización entre los pacientes con neoplasias hematológicas malignas, así como para reducir el riesgo de reacciones transfusionales hemolíticas. Por lo tanto, realizar fenotipado y genotipado para identificar sistemas distintos de ABO / Rh, especialmente para pacientes con transfusión crónica con síndrome mielodisplásico, puede minimizar el riesgo de aloinmunización y sus consecuencias, con efectos positivos sobre la seguridad y eficacia de la terapia transfusional.

Palabras clave: Aloinmunización; Anticuerpo irregular; Fenotipado de eritrocitos; Enfermedades oncohematológicas; Pacientes multitransfundidos.

\section{Introdução}

A medicina transfusional, enquanto conhecimento e prática médica, busca tornar-se cada vez mais segura para os pacientes cuja condição clínica demanda a terapia com componentes sanguíneos, a chamada hemoterapia (Adati, et al., 2009). Portanto, as boas práticas e a vigilância são asseguradas em todo ciclo do sangue (García-Roa, et al., 2017).

Nos hospitais e/ou em serviços de hemoterapia a transfusão sanguínea é amplamente utilizada, mas expõe o paciente à aloimunização pelo contato com vários antígenos estranhos, que são imunógenos potenciais, e que podem levar ao desenvolvimento de anticorpos no receptor dentro de dias, semanas ou meses após a transfusão (Alves, et al., 2012). Nos últimos anos, o principal objetivo das pesquisas sobre terapia transfusional tem sido reduzir o risco de aloimunização nos pacientes, uma vez que este processo promove alterações importantes no equilíbrio do sistema imunológico, já que o estímulo antigênico na população politransfundida é constante (Molina-Aguilar, et al., 2020). Em pacientes aloimunizados observa-se que a resposta imune humoral é estabelecida com redução significativa da produção de linfócitos $\mathrm{T}^{\mathrm{CD}} 4^{+}$, aumento de linfócitos $\mathrm{TCD}^{+}$, aumento de linfócitos B e deficiência de linfócitos T reguladores (Treg) (Molina-Aguilar, et al., 2020). Além disso, a aloimunização torna o paciente predisposto a reações transfusionais e dificulta a seleção de hemocomponentes compatíveis (Ido \& Oliveira, 2020; Molina-Aguilar, et al., 2020).

O risco de desenvolvimento de aloanticorpos depende de fatores como o número e frequência de transfusões, gravidez, imunogenicidade do antígeno, resposta imune do receptor, etnia do paciente e diferença no padrão antigênico do doador e do receptor (Kim, et al., 2019; Mangwana, et al., 2019).

As reações transfusionais são eventos adversos podendo ser classificados em reações alérgicas brandas, com surgimento de erupção cutânea, prurido, urticária; reações alérgicas graves com repercussão cardiovascular e respiratória; reações hemolíticas agudas, que ocorrem dentro de $24 \mathrm{~h}$ após a transfusão, e podem ser: 1) imuno-mediadas, que ocorre em resposta aos antígenos eritrocitários levando a hemólise intra ou extravascular com um quadro que evolui rápido e pode levar ao óbito; 2) não-imunes, em que a hemólise decorre de outros fatores (armazenamento dos hemocomponentes ou processo de infusão); reações hemolíticas tardias, que ocorrem 48h após a transfusão devido a reexposição a antígenos eritrocitários causando subsequente hemólise eritrocitária; reações febris não-hemolíticas, relacionadas a citocinas pró-inflamatórias ou à interação antígeno-anticorpo; sobrecarga circulatória relacionada à transfusão sanguínea; e Lesão Pulmonar Aguda Associada à 
Transfusão (TRALI), que se caracteriza pelo desenvolvimento de edema pulmonar não cardiogênico após a transfusão (Delaney, et al., 2016; Frazier, et al.,2017). Qualquer reação transfusional adversa se torna um agravo importante em pacientes aloimunizados.

Desde a descoberta do grupo sanguíneo ABO por Karl Landsteiner em 1900 e o desenvolvimento da técnica de antiglobulina indireta por Coombs, Mourant e Race em 1945, houve uma acentuada redução em fatalidades associadas à transfusão de sangue e a identificação de vários anticorpos antieritrocitários (Handa, et al., 2020). A Sociedade Internacional de Transfusão Sanguínea (International Society ofBloodTransfusion, ISBT) já reconheceu cerca de 360 antígenos de grupos sanguíneos, dos quais 326 estão caracterizados e organizados em 39 sistemas de grupos sanguíneos (Storry, et al., 2019). Esses sistemas são estudados devido à sua importância na medicina transfusional, sendo o ABO, Rh, MNS, Kell, Duffy, Kidd e Diego os mais imunogênicos e envolvidos em reações transfusionais (Daniels \& Reid, 2010; Ido \& Oliveira, 2020).

Na prática transfusional, um dos grupos de pacientes que requerem maior demanda por hemocomponentes são os que apresentam neoplasias, hematológicas ou não. Entre esses indivíduos a anemia é frequente e possui diversas etiologias como infiltração medular, sobrevida curta das células sanguíneas, hemorragia, supressão da resposta a eritropoetina, resposta imune comprometida (Abo el Fetouh, 2020). Por ser uma condição comum manifestada por pacientes onco-hematológicos, a queda constante na concentração de hemoglobina torna recorrente a terapia transfusional a fim de aumentar o aporte de oxigênio para os tecidos (Evers, et al., 2017).

De acordo com a literatura, dentre as doenças onco-hematológicas mais prevalentes no grupo de pacientes politransfundidos, destacam-se as Síndromes Mielodisplásicas (SMD) e as leucemias agudas (Evers, et al., 2017; Abo el Fetouh, 2020). As SMD compreendem um grupo de doenças clonais manifestadas por citopenia periférica associada à hiperplasia medular e alto risco de transformação para Leucemia Mieloide Aguda (LMA) (Rozovski, et al., 2015; Leisch, et al., 2017; Moncharmont, et al., 2019). As leucemias, grupo de neoplasias malignas do tecido hematopoiético, sãoclassificadas em agudas e crônicas, e em mieloide ou linfoide (Moraes, et al., 2017). As leucemias agudas são mais agressivas, com curto tempo de instalação e se caracterizam pelo predomínio de blastos no sangue periférico e na medula óssea, uma vez que a célula leucêmica perde a capacidade de diferenciação (Lamego, et al.,2010; Moraes, et al., 2017).As leucemias crônicas, de instalação insidiosa, preservam certa capacidade de diferenciação celular (morfológica), mas não funcional, com hiperproliferação de determinada linhagem celular, a depender da doença (Yamamoto \& Figueiredo, 2005; Hamerschlak, 2008). Pacientes que apresentam SMD ou leucemias geralmente desenvolvem múltiplos aloanticorpos (Rozovski, et al., 2015; Singhal, et al., 2017).

Como pacientes politransfundidos portadores de doenças onco-hematológicas apresentam elevado risco de aloimunização, esta revisão tem o intuito de fornecer informações importantes sobre o perigo da exposição cumulativa aos eritrócitos. Adicionalmente, verificar quais são os aloanticorpos antieritrocitáriosmais comuns na população de estudo e avaliar quais são as doenças onco-hematológicas mais frequentes.

\section{Metodologia}

Trata-se de uma revisão de literatura, utilizando como modelo as recomendações propostas pelo Preferred Reporting Items for Systematic Reviews and Meta-Analyses (PRISMA) (Moher, et al., 2009). A pergunta de pesquisa foi elaborada de acordo com a estratégia PCC (População, Conceito e Contexto) (Araújo, 2020) e ficou estruturada como: quais as neoplasias e os aloanticorpos mais comuns identificados em pacientes com doença onco-hematológica aloimunizados por politransfusão?

\subsection{Critérios de elegibilidade}

Foram incluídos neste trabalho estudos observacionais que envolveram pacientes politransfundidos com doenças 
onco-hematológicas e que desenvolveram aloimunização entre o período de 2010 a 2020, indexados na base de dados da United States National Library of Medicine (PUBMED), com idioma em inglês ou português, e grupo etário de adultos, maiores de 18 anos.

Foram excluídos trabalhos com gestantes, estudos com pacientes que desenvolveram apenas autoanticorpos, estudos que discutiram sobre doenças hematológicas como as hemoglobinopatias hereditárias (doença falciforme e talassemia), artigos com modelos animais, artigos não indexados, indisponíveis para download gratuito, dissertações e teses, artigos com dados insuficientes e/ou incompletos.

\subsection{Fontes de informação e estratégia de busca}

De acordo com os critérios de elegibilidade foram realizadas buscas na base de dados bibliográficos eletrônicos MEDLINE. Os termos de busca/descritores utilizados foram selecionados de acordo com o MeSH (Medical SubjectHeadings): "ErythrocyteTransfusion" and "HematologicDiseases".

A triagem dos manuscritos foi realizada a partir de títulos e resumos para excluir os estudos que não se encaixaram nos critérios de elegibilidade. Os artigos completos selecionados foram avaliados quanto aos critérios de inclusão e os seguintes dados extraídos: primeiro autor, ano de publicação, origem da população estudada, desenho do estudo, tamanho da amostra e tipo de doença, idade, quantidade e tipo de aloanticorpos detectados.

\section{Resultados}

A partir das buscas realizadas na base de dados PubMed foram identificadas 2.786 citações, de acordo com os termos de busca/descritores utilizados ("ErythrocyteTransfusion" and "HematologicDiseases"). A partir do título e/ou resumo, foram selecionados 31 artigos completos, dos quais, respeitando todos os critérios de inclusão, 10 foram utilizados para compor esta revisão (Figura 1). 
Figura 1. Fluxograma representativo da seleção dos trabalhos utilizados na revisão.

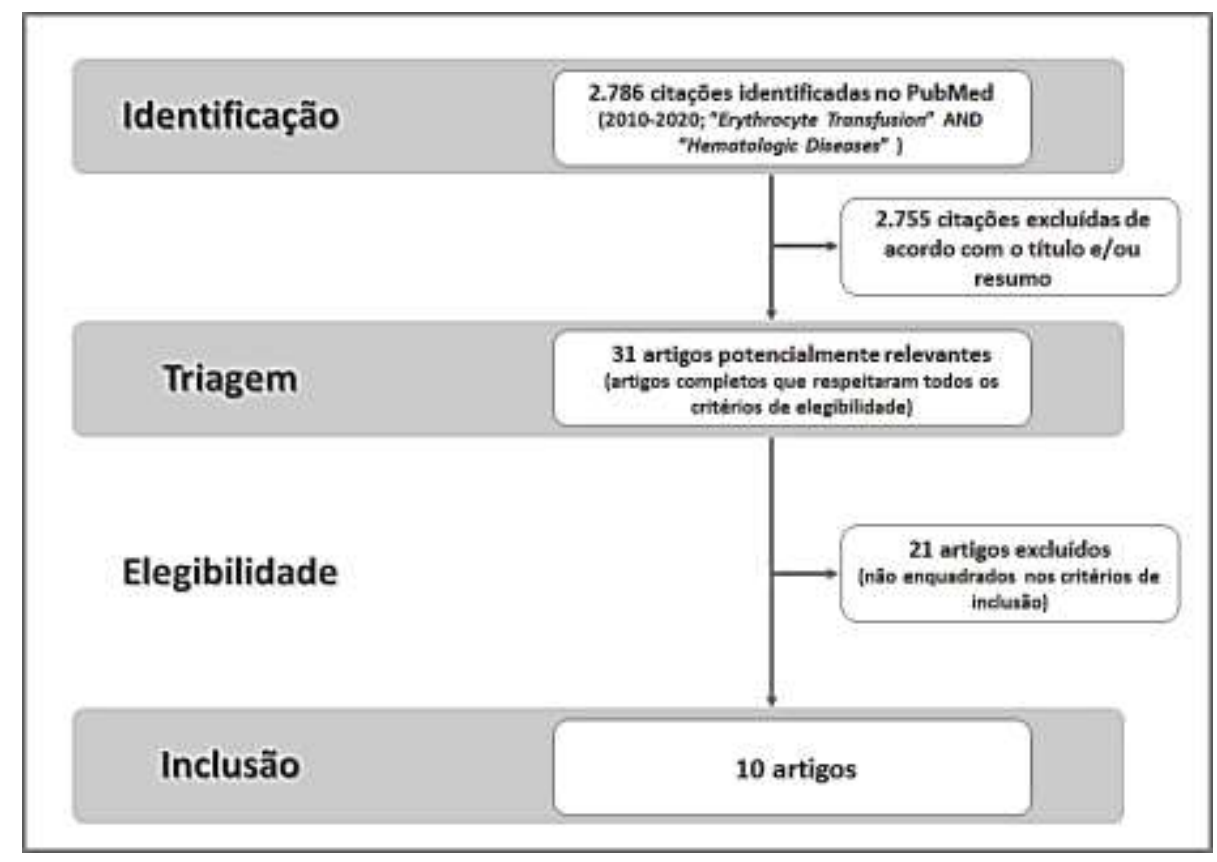

Fonte: Ramos, et al. (2021).

A Tabela 1 reúne os principais dados extraídos dos 10 artigos selecionados para compor este trabalho. São estudos observacionais (transversais e longitudinais) realizados em diferentes regiões do mundo (África, Ásia, Europa, América Latina). Entre as doenças hematológicas identificadas nos artigos, as mais comuns foram Síndrome Mielodisplásica, encontradas em 8 artigos, correspondendo a 80\% as leucemias, encontradas em 6 artigos, correspondendo a $60 \%$. A média de idade dos pacientes nos dez trabalhos analisados foi de 56 anos. Dentre os pacientes com SMD verificou-se uma média de idade superior a 60 anos. A maioria dos estudosidentificaram a presença dos seguintes aloanticorpos: anti-E (9/10; 90\%); antiK (7/10; 70\%); anti-S (6/10; 60\%); anti-Jk ${ }^{\mathrm{a}}(6 / 10 ; 60 \%)$; anti-c (5/10; 50\%); anti-M (5/10; 50\%); anti-Fy ${ }^{\mathrm{a}}(4 / 10 ; 40 \%)$, anti-C $(4 / 10 ; 40 \%)$ e anti-Fy ${ }^{\mathrm{b}}$ (3/10; 30\%),relacionados aos sistemas Rh, Kell, MNS, Kidd e Duffy. Finalmente, todos os trabalhos apontaram a fenotipagem eritrocitária extensa como forma de minimizar as taxas de aloimunização entre os pacientes com neoplasias hematológicas, assim como reduzir os riscos de ocorrência de reações hemolíticas transfusionais. 
Research, Society and Development, v. 10, n. 7, e26110716575, 2021

(CC BY 4.0) | ISSN 2525-3409 | DOI: http://dx.doi.org/10.33448/rsd-v10i7.16575

Tabela 1. Características e principais resultados dos estudos incluídos sobre aloimunização em pacientes politransfundidos com doença onco-hematológica.

\begin{tabular}{|c|c|c|c|c|c|c|c|}
\hline \multirow[t]{2}{*}{ Estudo } & \multirow[t]{2}{*}{ Origem } & \multirow{2}{*}{$\begin{array}{l}\text { Desenho do } \\
\text { estudo }\end{array}$} & \multirow{2}{*}{$\begin{array}{c}\text { Casos } \\
(\mathbf{N})\end{array}$} & \multirow[t]{2}{*}{ Doença } & \multirow[t]{2}{*}{ Idade $^{\#}$} & \multicolumn{2}{|c|}{ Aloanticorpos* } \\
\hline & & & & & & $\mathbf{N}(\%)$ & Tipo \\
\hline $\begin{array}{l}\text { Natukunda, et al., } \\
2010\end{array}$ & Uganda & Estudo transversal & 4 & $\begin{array}{c}\text { Neoplasias } \\
\text { hematológicas } \\
\text { NE }\end{array}$ & 34,8 & & $\begin{array}{l}\text { Anti-E } \\
\text { Anti-S } \\
\text { Anti-D (D parcial } \\
\left.\text { categoria } \mathrm{R}_{0}{ }^{\mathrm{HAR}}\right) \\
\text { Anti-K } \\
\text { Anti-Le } \\
\text { Anti-E e anti-K } \\
\text { Anticorpos pan } \\
\text { reativos }\end{array}$ \\
\hline Philip, et al., 2014 & Índia & $\begin{array}{l}\text { Estudo } \\
\text { transversal }\end{array}$ & 5 & $\begin{array}{l}\text { LMC (2) } \\
\text { SMD (3) }\end{array}$ & 48,5 & 2 & $\begin{array}{l}\text { Anti-c } \\
\text { Anti-S }\end{array}$ \\
\hline & & & & & 45,7 & 3 & $\begin{array}{l}\text { Anti-M } \\
\text { Anti-c } \\
\text { Anti-E }\end{array}$ \\
\hline $\begin{array}{l}\text { Caamaño, et al., } \\
2015\end{array}$ & Chile & $\begin{array}{l}\text { Estudo } \\
\text { longitudinal (caso- } \\
\text { controle) }\end{array}$ & 6 & $\begin{array}{l}\text { LLA } \\
\text { LNH } \\
\text { SMD } \\
\text { MM }\end{array}$ & 54 & & $\begin{array}{l}\text { Anti-E } \\
\text { Anti-K } \\
\text { Anti-D } \\
\text { Anti-Fy } \\
\text { Anti-c } \\
\text { Anti-Jk }^{\mathrm{a}} \\
\text { Anti-M } \\
\text { Anti-Le } \\
\text { Anti-Lu }^{\mathrm{b}} \\
\text { Anti-Js } \\
\text { Anti-s }\end{array}$ \\
\hline $\begin{array}{l}\text { Guelsin, et al., } \\
2015\end{array}$ & Brasil & $\begin{array}{l}\text { Estudo } \\
\text { transversal }\end{array}$ & 19 & SMD & 64 & $\begin{array}{l}2 \\
6 \\
1 \\
1 \\
1 \\
1 \\
1 \\
1\end{array}$ & $\begin{array}{l}\text { Anti-C } \\
\text { Anti-E } \\
\text { Anti-C }{ }^{w} \\
\text { Anti-S } \\
\text { Anti-Dia } \\
\text { Anti-Lu }^{\text {a }} \\
\text { Anti-C, anti-E } \\
\text { Anti-C, anti-e }\end{array}$ \\
\hline
\end{tabular}




\begin{tabular}{|c|c|c|c|c|c|c|c|}
\hline & & & & & & $\begin{array}{l}1 \\
1 \\
1 \\
1 \\
1\end{array}$ & $\begin{array}{l}\text { Anti-c, anti-E } \\
\text { Anti-c, anti-Jk } \\
\text { Anti-E, anti-K } \\
\text { Anti-C, anti-K, } \\
\text { anti-Di }{ }^{\mathrm{a}} \\
\text { Anti-E, anti-Jk }^{\mathrm{a}} \text {, } \\
\text { anti-Di }^{\mathrm{a}}\end{array}$ \\
\hline $\begin{array}{l}\text { Rozovski, et al., } \\
2015\end{array}$ & Israel & Estudo transversal & 18 & SMD & 76 & $\begin{array}{l}7 \\
5 \\
2 \\
2\end{array}$ & $\begin{array}{l}\text { Anti-K } \\
\text { Anti-E } \\
\text { Anti-Kp } \\
\text { Anti- } \mathrm{Lu}^{\mathrm{a}}\end{array}$ \\
\hline \multirow[t]{3}{*}{ Baía, et al., 2016} & \multirow[t]{3}{*}{ Portugal } & \multirow[t]{3}{*}{ Estudo transversal } & \multirow[t]{3}{*}{12} & LA (1) & 54 & 1 & Anti- Jk ${ }^{\mathrm{a}}$ \\
\hline & & & & $\operatorname{SMD}(6)$ & 80 & $4 ; 1 ; 1$ & $\begin{array}{l}\text { Anti -E; anti-K; } \\
\text { Anti- } \\
\text { (fenotipado) }\end{array}$ \\
\hline & & & & DMP (5) & 72 & $2 ; 1 ; 1 ; 1$ & $\begin{array}{l}\text { Anti-K; anti-C; } \\
\text { Anti- } \quad \mathrm{Jk}^{\mathrm{a}} \\
\text { (fenotipado); } \\
\text { Anti-Fy } \\
\text { (fenotipado) }\end{array}$ \\
\hline Kim, et al., 2019 & $\begin{array}{l}\text { Coréia } \\
\text { do Sul }\end{array}$ & Estudo transversal & 5 & SMD & 69,2 & $\begin{array}{l}1 \\
1 \\
1 \\
1 \\
1\end{array}$ & $\begin{array}{l}\text { Anti-E } \\
\text { Anti-E, anti-Jk } \\
\text { Anti-E, anti-c } \\
\text { Anti-E, anti-c, } \\
\text { anti-Fy } \\
\text { Anti-E }\end{array}$ \\
\hline Noor, et al., 2019 & Malásia & Estudo transversal & 7 & $\begin{array}{l}\text { LLA (3) } \\
\text { LNH (3) } \\
\text { MM (1) }\end{array}$ & 36,2 & $\begin{array}{l}4 \\
1 \\
1 \\
1\end{array}$ & $\begin{array}{l}\text { Anti-M, anti-E e } \\
\text { anti-S } \\
\text { Anti-E + anti-Le } \\
\text { Anti-E + anti-Le } \\
+ \text { anti-Mi } \\
\text { Anti-N }+ \text { anti-S }+ \\
\text { anti-Le }^{\mathrm{a}}+\text { anti- } \\
\text { Fy }^{\mathrm{a}}+\text { anti-Fy }^{\mathrm{a}}\end{array}$ \\
\hline $\begin{array}{l}\text { Molina-Aguilar, } \\
\text { et al., } 2020\end{array}$ & México & $\begin{array}{l}\text { Estudo } \\
\text { longitudinal }\end{array}$ & 19 & $\begin{array}{l}\text { SMD (12) } \\
\text { MM (5) }\end{array}$ & 55 & $\begin{array}{l}2 \\
3\end{array}$ & $\begin{array}{l}\text { Anti-S } \\
\text { Anti-K1 }\end{array}$ \\
\hline
\end{tabular}


Research, Society and Development, v. 10, n. 7, e26110716575, 2021

(CC BY 4.0) | ISSN 2525-3409 | DOI: http://dx.doi.org/10.33448/rsd-v10i7.16575

\begin{tabular}{|c|c|c|c|c|c|c|c|}
\hline & & (coorte) & & LMC (2) & & $\begin{array}{l}2 \\
4 \\
1 \\
1 \\
1 \\
1\end{array}$ & $\begin{array}{l}\text { Anti-I } \\
\text { Anti-Di }^{\mathrm{a}} \\
\text { Anti-e + anti-Jk } \\
\text { Anti-D } \\
\text { Anti-C } \\
\text { Anti-M }\end{array}$ \\
\hline $\begin{array}{l}\text { Abo el Fetouh, } \\
\text { et al., } 2020\end{array}$ & Egito & $\begin{array}{l}\text { Estudo } \\
\text { longitudinal } \\
\text { (coorte) }\end{array}$ & 83 & $\begin{array}{l}\text { LLA (22) } \\
\text { SMD (16) } \\
\text { LMA (13) } \\
\text { LLC (11) } \\
\text { LNH (10) } \\
\text { MM (7) } \\
\text { LMC (4) }\end{array}$ & 40,1 & $\begin{array}{c}25 \\
9 \\
30 \\
10 \\
12 \\
11 \\
14 \\
6 \\
7 \\
4 \\
3 \\
2 \\
1 \\
1\end{array}$ & $\begin{array}{l}\text { Anti-K } \\
\text { Anti-Kp } \\
\text { Anti-E } \\
\text { Anti-D } \\
\text { Anti-C } \\
\text { Anti-c } \\
\text { Anti-Fy } \\
\text { Anti-Fy } \\
\text { Anti-Jk }^{\mathrm{a}} \\
\text { Anti-Jk } \\
\text { Anti-M } \\
\text { Anti-N } \\
\text { Anti-S } \\
\text { Anti-Le }\end{array}$ \\
\hline
\end{tabular}

DMP; Desordem mieloproliferativa, LA; Leucemia aguda; LLA, Leucemia linfocítica aguda; LLC, Leucemia linfocítica crônica; LMA,

Leucemia mieloide aguda; LMC, Leucemia mieloide crônica; LNH, Linfoma não Hodgkin; SMD, Síndrome mielodisplásica; MM,

Mieloma múltiplo; NE, não especificadas.

*Em alguns trabalhos não houve a discriminação do número e/ou porcentagem de aloanticorpos detectados uma vez que os autores

Não especificaram quais destes anticorpos foram identificados especificamente nos pacientes com doença onco-hematológica.

"Média em anos. 


\section{Discussão}

A aloimunização causada pela incompatibilidade entre antígenos do doador e do receptor do hemocomponente desencadeia uma resposta imune de graus variados proporcionais à imunogenicidade e dose do(s) antígeno(s) incompatível(eis) (Evers, et al., 2017). As reações estão relacionadas a vários fatores, dentre eles idade, sexo e composição genética do paciente, presença de comorbidades, bem como o número e frequência de transfusões a que ele é submetido (Handa, et al., 2020). O desenvolvimento de anticorpos irregulares em pacientes politransfundidos é muito comum e o risco de aloimunização aumenta com o número de transfusões realizadas (Pimpaldara, et al., 2015; Celli, et al., 2017). Hemoglobinopatas, renais crônicos, pacientes com doenças onco-hematológicas e transplantados, apresentam taxa de aloimunização de mais de 50\% (Reyhaneh, et al., 2013).

A terapêutica transfusional é uma prática importante e comum nos pacientes portadores de doenças oncohematológicas como os diversos tipos de leucemias, linfomas e Síndromes Mieolodisplásicas (Singhal, et al., 2017). Ela tem como objetivo melhorar a capacidade de transporte de oxigênio e o fluxo de sangue na microcirculação (Federici, et al., 2012). A literatura é concordante em relação às transfusões, visto que a população politransfundida é mais exposta a diferentes antígenos eritrocitários e mais propensa a formar aloanticorpos isolados ou múltiplos (Mangwana, et al., 2019).

Os aloanticorpos variam de acordo com a diversidade genética e ocorrem em 0,3-2\% da população geral (Pahuja, et al., 2012; Hendrickson, et al., 2018). As taxas de aloimunização estão entre 0,009\% e 0,6\% em doadores saudáveis, 1,4\% e 4,24\% em indivíduos previamente transfundidos, $20 \%$ em pacientes com talassemia, 18,7\% em portadores de doença falciforme e 44\% em pacientes com doenças hematológicas malignas como as Síndromes Mielodisplásicas (Chao, et al., 2013; Sood, et al., 2013; Tiwari, et al., 2014; Guelsin, et al., 2015; Kangiwa, et al., 2015; Pessoni, et al., 2018).

Conforme apontam os estudos incluídos nesta revisão, a realidade na medicina transfusional difere muito devido a vários fatores, entre os quais se destacam as restrições técnicas e econômicas enfrentadas por vários deles, o número de doadores e a qualidade dos testes, além do planejamento para o manejo dos pacientes que receberão múltiplas transfusões, tendo em vista a dificuldade de encontrar hemocomponentes compatíveis para essa população. Dois trabalhos também enfatizaram a importância do histórico clínico de transfusões sanguíneas prévias e da manutenção e acompanhamento dessas informações para a população politransfundida (Nakunda, 2010; Philip, 2014).

Diante dos dados apresentados neste trabalho, ao analisar diferentes populações no mundo, verificou-se que em 8 dos 10 estudos, a SMD foi a doença mais prevalente entre a população, particularmente acima dos 60 anos.A prevalência da SMD entre idosos decorre de uma maior suscetibilidade dessa população, uma vez que o envelhecimento causa alterações na hematopoiesecomo redução na produção de células eritrocitárias e linfocíticas, assim como aumento na produção de células da linhagem mieloide, podendo gerar o aparecimento de diversas neoplasias hematológicas (Chung\&Park, 2017). Esse dado está consoante com informações encontradas na literatura, como em um estudo realizado na Austrália, com pacientes que desenvolveram mielodisplasia e apresentaram uma médiade 73 anos de idade (Singhal, et al., 2017), e estudos que apontam que esta é uma condição particularmente comum em pessoas idosas, juntamente com a leucemia mieloide aguda e leucemia mielomonocítica crônica (Leisch, et al., 2017).

Rozovski e colaboradores (2015) indicam que a SMD é o mais forte preditor para a formação de aloanticorpos durante a terapia transfusional, entretanto, apontam que ainda persistem dúvidas quanto ao fato destes pacientes apresentarem uma maior propensão para a aloimunização ocasionada pelas múltiplas transfusões, ou se a doença em si é um fator de risco para a aloimunização. Outro trabalho que correlacionou doenças linfoproliferativas com a produção de aloanticorpos apontou que em pacientes com Leucemia Linfoide Crônica há um efeito "protetor" ocasionado, provavelmente, pela substituição de linfócitos T e B funcionais por células clonais (Philip,et al., 2014). Além disso, existem relatos que a administração de corticosteroides e 
outras drogas imunossupressoras prejudicariam a resposta imune mediada por células $\mathrm{T}$ aos antígenos de doadores, reduzindo assim o risco de aloimunização (Zalpuri, et al., 2014). Evidentemente, a redução deste risco mediado pela terapia imunossupressora pode não ser a única explicação, mas seria uma causa plausível (Zalpuri, et al., 2014; Singhal, et al., 2017).

Dos aloanticorpos identificados, observou-se uma maior frequência de anticorpos contra antígenos do sistema Rh (C, c, E, e), especialmente o anti-E, relatado em 9 dos 10 artigos analisados. Anticorpos do sistema Kell também são considerados relevantes e foram relatados em 7 dos 10 artigos avaliados. Consoante com dados encontrados na literatura e considerando que esses aloanticorpos são os mais frequentes, seus antígenos relacionados também são apontadoscomo os mais imunogênicos (Alves, et al., 2012; Singhal, et al., 2017). Outros anticorpos também foram identificados (sistema MNS, anti-S e anti-M; sistema Kidd, anti-Jk ${ }^{\mathrm{a}}$ e sistema Duffy, anti-Fy ${ }^{\mathrm{a}}$ e anti-Fy ${ }^{\mathrm{b}}$ ) e puderam ser encontrados combinados em pacientes aloimunizados. O aparecimento e identificação de diferentes anticorpos de sistemas distintos demonstram a importância de uma fenotipagem estendida como forma de reduzir a aloimunização, principalmente em pacientes politransfundidos.

Atualmente uma alternativa disponível para a prevenção da aloimunização é a realização da fenotipagem eritrocitária dos pacientes receptores e das bolsas de sangue, a qual consiste em identificar quais antígenos estão presentes na superfície doeritrócito, uma vez que indivíduos que não possuem determinado antígeno podem produzir anticorpos frente a essa exposição (Baía, et al., 2016). Todos os estudos avaliados nesta revisão compartilham a ideia de que há a necessidade de uma compatibilização extensa dos antígenos eritrocitários do doador e do receptor, a fim de reduzir as taxas de imunização populacional e diminuir o número de reações hemolíticas transfusionais, além de aprimorar as práticas para aumentar a segurança e eficácia desta terapia.

A aloimunização pode ser prevenida ou reduzida, através da ampliação da fenotipagem e compatibilização para os antígenos C, c, E, e (sistema Rh) e K (sistema Kell), além do Fya (sistema Duffy), Jkª (sistema Kidd) e S (sistema MNS) para os pacientes cronicamente transfundidos. A presença destes antígenos foi evidenciada na maioria dos trabalhos incluídos neste estudo. Para pacientes com SMD, uma estratégia, além da fenotipagem, seria a genotipagem para antígenos do sistema Rh e Kell (Guelsin, et al., 2015), técnica precisa, de fácil execução e que apresenta viabilidade financeira, visto que preveniria a grande maioria dos efeitos adversos e permitiria o melhor uso dos hemocomponentes aos pacientes acometidos por essa doença e que necessitam de terapia transfusional recorrente.

\section{Considerações Finais}

Embora a associação entre a exposição a antígenos eritrocitários estranhos e a aloimunização seja conhecida há décadas, os fatores específicos que predispõe indivíduos politransfundidos a produzir aloanticorpos de forma distinta permanecem mal compreendidos, sobretudo quando relacionados a doenças importantes como as malignidades hematológicas.

A presente revisão reúne os dados extraídos de artigos recentes que tratam a respeito de aloimunização em pacientes politransfundidos com doença onco-hematológica, e conclui que a doença mais prevalente nesta população foi a Síndrome Mielodisplásica e que os aloanticorpos detectados com maior frequência foram os associados aos sistemas Rh, Kell, MNS e Kidd. Embora a realidade na medicina transfusional difira entre os países devido a muitos fatores, a realização da fenotipagem e genotipagem para a identificação de outros sistemas além do $\mathrm{ABO} / \mathrm{Rh}$, principalmente para os pacientes cronicamente transfundidos, pode minimizar o risco de aloimunização e suas consequências, aumentando a segurança e eficácia da terapia transfusional.

\section{Referências}

Abo el Fetouh, R. M., Abd Elmoniem, G. M., Allam, R. M., Sobeih, M. E., Kamel M. M., \& Radwan, S. M. (2020). Frequency and specificity of Red blood cell alloantibodies in multitransfused Egyptian patients with hematological and non hematological malignancies. Transfusion and Apheresis Science, 59(6), 15. 
Adati, M. C., Gemal, A. L., \& Guedes, H. C. B. (2009). Resultados do controle de qualidade de produtos hemoderivados: análise sanitária. Rev Bras Hematol Hemoter, 31(4), 235-40.

Alves, V. M., Martins, P. R. J., Soares, S., Araujo, G., Schmidt, L. C., \& Costa, S. S. M. (2012). Pesquisa de aloimunização após transfusão de concentrados de hemácias em um estudo prospectivo. Rev Bras Hematol Hemoter, 34(3), 206-11.

Araujo, W.C. O. (2020). Recuperação da informação em saúde: construção, modelos e estratégias. Conv Ciênc Inform, 3(2), 100-34.

Baia, F., Correia, F., Alves, B., Martinez, F., Koch, C., \& Carneiro, A. (2016). Phenotyping Rh/Kell and risk of alloimmunization in haematological patients. Transfus Med, 26(1), 34-8.

Caamaño, J., Musante, E., Contreras, M., Ulloa, H., Reyes, C., \& Inaipil, V. (2015). Frequency and specificity of red blood cell alloimmunization in chile an transfused patients. Transfus Med Hemother, 42(1), 4-7.

Celli, R., Schulz, W., Hendrickson, J. E., \& Torney, C. A. (2017). A novel network analysis tool to identify relationships between disease states and risks for red blood cell alloimmunization. Vox Sang, 112(5), 469-72.

Chao, Y. H., Wu, K. H., Lu, J. J., Shih, M. C., Peng, C. T., \& Chang, C. W. (2013). Red blood cell alloimmunization among Chinese patients with $\beta$ thalassaemia major in Taiwan. BloodTransfus, 11(1), 71-4.

Chung, S. S., \& Park, C. Y. (2017). Aging, hematopoiesis, and the myelodysplastic syndromes. Hematology Am Soc Hematol Educ Program, 2017(1), 73-8.

Daniels, G., \& Reid, M. E. (2010). Blood groups: the past 50 years. Transfusion, 50(2), 281-9.

Delaney, M. D., Wendel, S., Bercovitz, R. S., Cid, J., Cohn, C., \& Dunbar, N. M. D. (2016). Transfusion reactions: prevention, diagnosis, and treatment. The Lancet, 388(10061), 1-12.

Evers, D., Zwaginga, J. J., Tijmensen, J., Middelburg, R. A., Haas, M., \& Vooght, K. M. K. (2017). Treatments for hematologic malignancies in contrast to those for solid cancers are associated with reduced red cell alloimmunization. Haematologica, 102(1), 52-9.

Frazier, S. K., Higgins, J., Bugajski, A., Jones, A. R., \& Brown, M. R. (2017). Adverse reactions to transfusion of blood products and best practices for prevention. Crit Care Nurs Clin North Am, 29(3), 271-90.

Federici, A. B., Vanelli, C., \& Arrigoni, L. (2012).Transfusion issues in cancer patients. Thrombosis Research, 129(1), 60-5.

García-Roa, M.,Vicente-Ayuso, M. D. C., Bobes, A. M., Pedraza, A. C., Gonzalez-Fernandez, A., \& Martin, M. P. (2017). Red blood cell storage time and transfusion: current practice, concerns and future perspectives. Bloodtransfus, 15(3), 222-31.

Guelsin, G. A. S., Rodrigues, C., Visentainer, J. E. L., Campos, P. M., Traina, F., \& Gilli, S. C. O. (2015). Molecular matching for Rh and K reduces red blood cell alloimmunization in patients with myelodisplastic syndrome. Blood Transfus, 13(1), 53-8.

Hamerschlack, N. (2008). Leucemia: fatores prognósticos e genética. J Pediatr, 84(4), 52-7.

Handa, A.,Kukar, N., Maharishi, R. N.,Syal, N., \& Arora, H. (2020). Analysis of red cell alloimmunization in multi transfused patients at a Tertiary care teaching hospital. J Family Med Prim Care, 9(6), 2907-11.

Hendrickson, J. E., \& Tormey, C. A. (2016). Understanding red blood cell alloimmunization triggers. Hematology Am Soc Hematol Educ Program,2016(1), 446-51.

Ido, A. A. S., \& Oliveira, M. C. (2020). Main Erythrocyte Antigens Involved in the Alloimmunization Process. Open Science Journal, 5(2), 2-10.

Kangiwa, U., Ibegbulam, O., Ocheni, S., \& Madu, A., Mohammed, N. (2015). Pattern and prevalence of alloimmunization in multiply transfused patients with sickle cell disease in Nigeria. Biomark Res, 3(26).

Kim, H. Y., Cho, E. J., Chun, S., Kim, K. H., \& Cho, D. (2019). Red Blood Cell Alloimmunization in Korean Patients With Myelodysplastic Syndrome and Liver Cirrhosis. Ann labmed, 39(2), 218-22.

Lamego, R.M., Clementino, N. C. D., Costa, A. L. B., Oliveira, M. J., \& Bittencourt, H. (2010). Transplante alogênico de célu las-tronco hematopoéticas em leucemias agudas: a experiência de dez anos do Hospital das Clínicas da UFMG. Rev Bras Hematol Hemoter, 32(2), 108-15.

Leisch, M., Weiss, L., Lindlbauer, N., Jungbauer, C., Egle, A., \& Rohde, E. (2017). Red blood cell alloimmunization in 184 patients with myeloid neoplasms treated with azacitidine: A retrospective single center experience. Leuk Res,59, 12-19.

Mangwana, S.,Kacker, A., \& Simon, N. (2019). Red cell alloimmunization in multi-transfused, oncology patients: Risks and management. Glob J Transfus Med, 4(1), 74-8.

Moher, D., Liberati, A., Tetzlaff, J., \& Altman, D. G. (2009). Preferred reporting items for systematic reviews and meta-analyses: the PRISMA statement. PLoS Medicine, 6(7), 1-6.

Molina-Aguilar, R., Gómez-Ruiz, S., Vela-Ojeda, J., Montiel-Cervantes, L.A., \& Reyes-Maldonado, E. (2020).Pathophysiology of Alloimmunization. Transfus Med Hemother, 47, 152-9.

Moncharmont, P., Quittançon, E., Barday, G., \& Benamara, A. (2019). Adverse transfusion reactions in patients with aplastic anaemia or myelodysplastic syndromes. Vox Sang,114(4), 349-54. 
Research, Society and Development, v. 10, n. 7, e26110716575, 2021

(CC BY 4.0) | ISSN 2525-3409 | DOI: http://dx.doi.org/10.33448/rsd-v10i7.16575

Moraes, E. S., Mello, M. S. C., Nogueira, F. A. M., Otero, U. B., \& Carvalho, F. N. (2017). Análise de indivíduos com leucemia: limitações do sistema de vigilância de câncer. Ciênc saúde colet, 22(10), 3321-32.

Natukunda, B.,Schonewille, H., Vande Watering, L., \& Brand, A. (2010) Prevalence and specificities of red blood cell alloantibodies in transfused Ugandans with different diseases. Vox Sang, 98(2), 167-71.

Noor, N. H. M., Arifin, N., Hassan, M. N., \& Mustaffa, R. (2019). Red cell alloimmunization among haemato-oncologic patients in a teaching hospital in Malaysia. Sri Lanka Journal of Medicine, 28(1), 41-8.

Pahuja, S., Kushwaha, S., Sethi, N., Pujani, M., \& Jain, M. (2012). Screening of blood donors for erythrocyte alloantibodies. Hematol, 17(5), $302-5$.

Pessoni, L. L., Ferreira, M. A., Silva, J. C. R., \& Alcântara, K. C. (2018).Red blood cell alloimmunization among hospitalized patients: transfusion reactions and low alloantibody identification rate. Hematol Transfus Cell Ther, 40(4), 326-31.

Pimpaldara, R. P., Patel, A. C., Patel, J., Patel, S., Pandya, A. N., \& Wadhwani, S. (2015). A Study of Irregular Antibodies in 200 Multi-Transfused Patients. J Evol Med Dent Sci, 4(73), 12659-67.

Philip, J., Biswas, A. K., Hiregoudar, S., \& Kushwaba, N. (2014).Red Blood Cell Alloimmunization in Multitransfused Patients in a Tertiary Care Center in Western India. Lab Med, 45(4), 324-30.

Reyhaneh, K., Ahmad, G., Gharib, K., Vida, V., Raheleh, K., \& Mehdi, T. N. (2013). Frequency and specificity of RBC alloantibodies in patients due for surgery in Iran. Indian J Med Res, 138(2), 252-6.

Rozovski, U., Ben-Tal, O., Kirgner, I., Mittelman, M., \& Hareuveni, M. (2015). Increased Incidence of Red Blood Cell Alloantibodies in Myelodysplastic Syndrome. Isr Med Assoc J, 17(10), 624-7.

Singhal, D., Kutyna, M. M., Chhetri, R., Wee, L. Y. A., Hague, S., \& Nath, L. (2017). Red cell alloimmunization is associated with development of autoantibodies and increased red cell transfusion requirements in myelodysplastic syndrome. Haematologica, 102(12), 2021-9.

Sood, R., Riana, V., \& Rosamma, N. L. (2013). Detection of alloimmunization to ensure safer transfusion practice. Asian J Transfus Sci, 7(2), 135-9.

Storry, J. R., Clausen, F. B., Castilho, L., Chen, Q., Daniels, G., \& Denomme, G. (2019). International Society of Blood Transfusion working party on red cell immunogenetics and blood group terminology: Report of the Dubai, Copenhagen and Toronto meetings. Vox Sang, 114(1), 95-102.

Tiwari, A. K., Pandey, P., Sharma, J., Shailja, K., Dixit, S., \& Raina, V. (2014). Incidence of clinically significant antibodies in patients and healthy blood donors: a prospective cross-sectional study from a tertiary healthcare center in India. Transfusion and Apheresis Science, 50(2), 230-4.

Yamamoto, M.; \& Figueiredo, V. L. P. (2005). Epidemiologia da leucemia linfocítica crônica e leucemia linfocítica crônica familiar. Rev Bras Hematol Hemoter, 27(4), 229-32.

Zalpuri, S., Evers, D., Zwaginga, J. J., Schonewille, H., Vooght, K. M. K., \& Cessie, S. (2014). Immunosuppressants and alloimmunization against red blood cell transfusions. Transfusion, 54(8), 1981-7, 2014. 